Referencia para citar este artículo: Parra-Valencia, L., Aponte-Muñoz, A. C., \& Dueñas-Manrique, M. M. (2018). Jóvenes, grupo y arte: las personas jóvenes y el arte re-unidos. Revista Latinoamericana de Ciencias Sociales, Niñez y Juventud, 16(2), 853-865. doi:https://doi.org/10.11600/1692715x.16214

\title{
Jóvenes, grupo y arte: las personas jóvenes y el arte re-unidos*
}

\author{
LILIANA PARRA-VALENCIA ** \\ Profesora Universidad Cooperativa de Colombia, Colombia. \\ Angie Carolina Aponte-Muñoz ${ }^{* * *}$ \\ Auxiliar de investigación Universidad Cooperativa de Colombia, Colombia. \\ Martha Milena DUEÑAS-ManRIQUE ${ }^{* * * *}$ \\ Auxiliar de investigación Universidad Cooperativa de Colombia, Colombia.
}

\section{Artículo recibido en septiembre 4 de 2017; artículo aceptado en diciembre 5 de 2017 (Eds.)}

- Resumen (descriptivo): analizamos prácticas grupales que sanan y aportan a los procesos de reconfiguración psicosocial y cambio social. Presentamos los resultados de investigación, desde una perspectiva interpretativa, de la relación entre jóvenes, grupo y arte. En particular, reflexionamos en torno a lo grupal y el arte contemporáneo en la reconfiguración de experiencias en jóvenes. Apuntalamos la metodología en la perspectiva cualitativa, por el interés en los significados de la realidad desde los sujetos. Incluimos la observación de la iniciativa juvenil Laboratorios por la Paz (Suba-Bogotá), inspirada en la técnica psicoanalítica poskleiniana Observación de bebés. La estrategia de análisis corresponde a la hermenéutica-interpretativa.El artículo concluye que ante la estigmatización que experimentan las personas jóvenes en Colombia, el grupo y el arte se configuran en estrategias de apoyo y resignificación emocional.

Palabras clave: acción sociocultural, arte contemporáneo, cambio social, conflicto armado, participación juvenil y psicología social (Tesauro de Ciencias Sociales de la Unesco).

\section{Youth, group and art. Young people and art re-united}

Abstract (descriptive): We analyze group practices that heal and contribute to the processes of psychosocial reconfiguration and social change. In this opportunity, we present the results of research from an interpretive perspective, the relationship between youth, group and art. In particular, we reflect on the group and the contemporary art in the reconfiguration of experiences in young people. The methodology was underpinned in the qualitative perspective, by the interest in the meanings of

Este artículo de reflexión es derivado de la investigación Psicología y grupalidad, que hace parte de un estudio mayor, en la línea de investigación Psicología e iniciativas sociales de paz en Colombia. Acta № 2016-1650 del 24 de febrero de 2016, del Comité Nacional de Investigación (Conadi) de la Universidad Cooperativa de Colombia. Realizado entre febrero de 2016 y agosto de 2017. Investigación de tipo cualitativa y perspectiva hermenéutica. Gran área: Ciencias Sociales; área de conocimiento: Psicología; subárea: temas sociales, interdisciplinaria.

** Psicóloga e investigadora social-comunitaria. Candidata al Doctorado en Ciencias Sociales y Humanas (Universidad Javeriana). Maestría en Psicología Social y Violencia Política (Universidad San Carlos de Guatemala). Maestría en Humanidades y Sociedades del siglo XXI (Universidad de Barcelona). Profesora del Programa de Psicología de la Universidad Cooperativa de Colombia (Bogotá). Investigadora principal de la línea Psicología e iniciativas sociales de paz en Colombia. Orcid: 0000-0002-9411-4513. Índice H5: 2. Correo electrónico: liliana.parrav@campusucc. edu.co

*** Psicóloga. Auxiliar de la investigación Psicología e iniciativas sociales de paz en Colombia. Universidad Cooperativa de Colombia (Bogotá). Orcid: 0000-0001-9168-0176. Correo electrónico: karola-5691@hotmail.com

**** Psicóloga. Auxiliar de la investigación Psicología e iniciativas sociales de paz en Colombia. Universidad Cooperativa de Colombia (Bogotá). Orcid: 0000-0003-3838-5455. Correo electrónico: mtmilena125@hotmail.com 
reality from the subjects. It included the observation of the Juvenile Initiative Laboratories for Peace (Suba-Bogotá), inspired by the postkleinian psychoanalytic observation of babies. The analysis strategy was hermeneutic-interpretive. The article concludes that before the stigmatization that the young people in Colombia experience, the group and the art are configured in strategies of support and emotional resignification.

Key words: Socio-cultural action, contemporary art, social change, armed conflict, youth participation and social psychology (Unesco Social Sciences Thesaurus).

\section{Jovem, grupo e arte. Os jóvens e o arte re-unidos}

- $\quad$ Resumo (descritivo): analizamos práticas grupales que sanan e aportan a processos de reconfiguração psicosocial e cambio social. Nesta oportunidade, apresenta os resultados de investigação de uma perspectiva interpretativa, da relação entre jóvens, grupo e arte. Em particular, reflexionamos no torno e o grupal e o arte contemporâneo na reconfiguração de experiências em jóvens. A metodologia se apuntaló na perspectiva qualitativa, pelo interesse nos significados da realidade desde os sujeitos. Incluíram a observação da iniciativa juvenil Laboratorios por a paz (Suba-Bogotá), inspirada na técnica psicoanalítica poskleiniana Observação de bebés. A estratégia de análise foi hermenéutica-interpretativa. $O$ artigo concluye que ante a estigmatização que experimentam os jóvens na Colômbia, o grupo e a arte se configura em estratégias de apoio e resignificação emocional.

Palavras-chave: Acção sociocultural, arte contemporânea, cambio social, conflito armado, participação juvenil e psicológica social (Tesauro de Ciências Sociais da Unesco).

-1. Introducción. Construyendo caminos para acercarnos a las personas jóvenes y a las expresiones artísticas. -2. Grupo y jóvenes. -3. Arte y jóvenes. -4. Metodología. Una ruta para estudiar el ámbito grupal juvenil. -5. Discusión. -6. Conclusiones. -Lista de referencias.

Figura 1. Grafiti realizado por las personas jóvenes del grupo Beat Flow Music (La BFM). Foro Jóvenes, grupo y arte. Universidad Cooperativa de Colombia. Mayo 7 de 2016

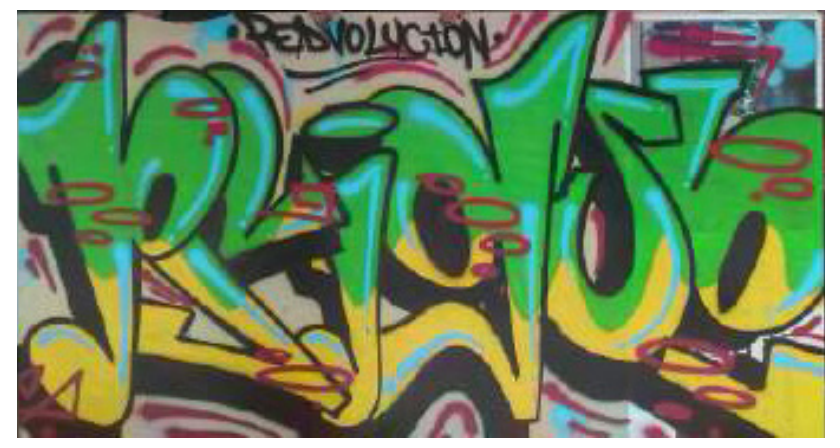

\section{Introducción. Construyendo caminos para acercarnos a las personas jóvenes y a las expresiones artísticas}

El interés por el estudio de los individuos jóvenes, de reciente data, ha pasado por diferentes momentos. Desde la perspectiva psicológica el sujeto joven se ha concebido como quien adolece de algo, en cuanto se vincula con la adolescencia. Esta mirada venida de la influencia biomédica, fue adoptada por la psicología más hegemónica. También, se ha concebido al individuo joven como aquel que atraviesa una etapa de la vida intermedia entre la infancia y la adultez; así que, la juventud es el periodo de transición donde la persona se prepara para asumir las responsabilidades de la vida adulta. 
En la actualidad cada vez más, las jóvenes y los jóvenes tienen que asumir la adultez de manera más prematura; en parte porque se los involucra al mercado, o por la necesidad de que aporten un ingreso a su familia e incluso para que asuman su propia manutención y formación educativa.

La manera de concebir a las personas jóvenes en Colombia, también ha estado permeada por una tendencia a vincularlas con la violencia. Esta percepción se generalizó en un momento dado a finales de la década del noventa del siglo XX, cuando su participación en bandas juveniles daba cuenta de ciertas problemáticas sociales como la delincuencia, el narcotráfico, el conflicto armado. Valga señalar que, al recrudecimiento de la violencia en esa década y final de milenio, se le sumó el posicionamiento militar y político del paramilitarismo en el país (Cinep \& Justicia y Paz, 1998), otro escenario que vinculó a los sujetos jóvenes a la maquinaria de la sangrienta guerra, en particular a quienes sufrían condiciones de vida precaria.

Ahora bien, a la hora de revisar la producción académica en el campo de la disciplina psicológica sobre jóvenes y conflicto armado, identificamos una tendencia a enfatizar en el impacto de este en la juventud. Los distintos estudios, en su mayoría, giran en torno a visibilizar el efecto emocional y psicosocial desencadenado en los individuos y las comunidades, a partir de un determinado hecho de violencia. Es decir, dichos estudios dan cuenta de manera reiterada de cómo la violencia y el conflicto armado en Colombia son vistos como generadores de alteraciones psicologías y emocionales en los individuos jóvenes, afectando su interacción social y deteriorando el vínculo con el otro.

Así pues, pareciera que es difícil advertir otra manera de concebir a los sujetos jóvenes, dado el énfasis de las políticas públicas y los programas institucionales en atender a la población joven solo desde la perspectiva de los efectos y consecuencias del conflicto armado.

Por tanto, nos interesan aquellas perspectivas que conciben a los individuos jóvenes como aquellos agentes que problematizan las realidades sociales, políticas y económicas, es decir, que potencian la sociedad con sus ideas de transformación.

En nuestro modo de acercarnos a las personas jóvenes, este énfasis de la mirada patologizante carga y limita a la población joven, en relación con el fracaso o el éxito en el afrontamiento de las problemáticas sociales que genera la guerra.

Más aún, se deja de lado el hecho de que la mayoría de las formas organizativas de las jóvenes y los jóvenes son autónomas y no dependen de propuestas estatales o gubernamentales para existir, dado que estos conforman grupos e impulsan iniciativas sociales de paz (Parra-Valencia, 2014), desde sus territorios de base. La gente joven se apropia de mecanismos para enfrentar las consecuencias, las violencias y la estigmatización, de una forma creativa que los protege de vincularse a lógicas bélicas. La juventud en este escenario nos invita a seguir creyendo que sí existe otro camino.

Teniendo en cuenta lo anterior, y para abordar la relación de los individuos jóvenes con lo grupal y lo artístico, en la primera parte del artículo proponemos la base teórica de nuestra investigación; esta privilegia la perspectiva psicológica del grupo, específicamente el lugar del grupo en el psiquismo individual y colectivo de la gente joven. Aquí nos apoyamos en los aportes que argumentan de qué manera las personas pueden cambiar su situación al compartir sus experiencias con otros (Beristain \& Riera, 1993), incluso en situaciones de violencia por guerra.

La mayoría de los estudios (Becker, Morales, \& Aguilar, 1994; López, 2012; Romero, 2012), tanto en Colombia como en otros países de Latinoamérica, convergen en que el grupo puede llegar a entenderse como una familia, que se convierte en el vehículo de transformación de los jóvenes y las jóvenes.

Ahora bien, el interés por la experiencia artística, en particular desde el trabajo de reconfiguración psíquica y psicosocial de las personas jóvenes en el ámbito local y comunitario, también hace parte de nuestra indagación. Nos ocupamos de la dimensión creativa en cuanto despliegue de formas de expresión, donde el arte cura (Rolnik, 2001). Así que dedicamos un apartado a la relación entre jóvenes y arte. 
La metodología se basó en la perspectiva cualitativa; la técnica de aproximación a la iniciativa juvenil fue la observación, en particular desde las ideas de la observación psicoanalítica de bebés, y la estrategia de análisis fue de tipo hermenéutico.

También planteamos las categorías que emergieron sobre la relación jóvenes, grupo y arte, siguiendo el procedimiento de la construcción categorial de la Teoría Fundamentada - codificación abierta, relacional y nuclear de la información- . Las categorías de estudio construidas dan cuenta del potencial del grupo y el valor del recurso artístico en la reconfiguración psíquica y social, en cuanto a la capacidad para sanar (Parra-Valencia, 2016). También se resaltan las acciones de resistencia y su relación con los procesos de memoria.

Finalmente, mencionamos algunas reflexiones a manera de conclusión.

\section{Grupo y jóvenes}

Nuestra indagación teórica nos llevó a preguntamos, ¿qué importancia tiene el grupo para las personas jóvenes?; para su psiquismo, tanto en la dimensión individual como en la colectiva. Lo primero, desde una mirada de la subjetividad; y lo segundo, desde un interés por resaltar la perspectiva emancipadora.

La investigación sobre jóvenes y grupo nos ayudó a identificar cómo el individuo joven genera procesos de transformación al pertenecer, de forma activa, a un colectivo, a una iniciativa o a una organización juvenil, teniendo en cuenta que la conformación de un grupo conlleva la construcción de una identidad colectiva, como lo menciona Carlos Martín Beristain.

El potencial del grupo está en desarrollar el «nosotros». Cuando unas personas se sienten identificadas con las otras, los lazos del grupo, hacen que tenga un gran potencial de ayuda mutua y transformación social (pasando del yo, o mi problema, al nosotros y nuestros problemas (Beristain \& Riera, 1993, p. 92).

En este sentido, del potencial de la ayuda mutua y el cambio, el psicólogo colombiano Manuel Eduardo López (Torres et al., 2012) da cuenta, en el caso de la Red Uniendo Sueños de la ciudad de Medellín, de las razones de agrupamiento alrededor de la Red: la consolidación de un proceso organizativo, el lugar de sus integrantes y la compresión de lo que es su grupo. Estos procesos, afirma López, permiten a las personas jóvenes no solamente protegerse entre sí, sino elaborar sus reacciones de adaptación y de estrés psicosocial.

Esta experiencia resalta la reconfiguración emocional que se gesta cuando la joven o el joven comparte, con los otros en grupo, sueños, objetivos e ideales, aún en situaciones de pobreza, exclusión y estigmatización.

Retomando los aportes de Beristain (2010), quien identifica que la capacidad de apoyo mutuo, entendida como la capacidad de controlar en conjunto fuerzas y reacciones impulsivas, se construye en el grupo, hay que decir que es allí donde es posible compartir experiencias y plantear soluciones a los problemas de manera colectiva, lo que proporciona una base para la reconstrucción.

En el caso de los individuos jóvenes, el grupo es fundamental en el proceso de identificaciones que experimenta con otros en la expansión de la socialización, en particular porque ya no solo hace parte del grupo familiar primario como tal, sino que es el momento para participar de grupos de pares, grupos de interés, entre otros.

Este primer grupo cumple una importante función de mediación entre sus propias fantasías y el mundo exterior. A partir de estas vivencias tempranas, se sientan las bases para participar a lo largo de la vida de otros grupos; allí emergen situaciones en las que se sitúan los miembros de los grupos de los que hacemos parte, en determinado rol que evoca a las figuras parentales de la infancia (Grinberg, Langer, \& Rodrigué, 1974). Ahora bien, la postura que asume cada uno de los miembros del grupo es fundamental en la interacción con los otros, ya que cualquier tipo de cambio se refleja en el grupo $\mathrm{y}$ en sus integrantes. 
Así es que, para las jóvenes y los jóvenes el grupo adquiere una relevancia significativa, y más en contextos de conflicto, ya que, como menciona Romero (2012), las personas jóvenes, al pertenecer a un grupo, pasan por cambios en su psiquismo, es decir, se genera una transformación por el grupo. Cada integrante se convierte en el continente-contenido (Bion, 1997) del otro cuando los sujetos jóvenes se reúnen.

En otro sentido, y conocedoras de los efectos terapéuticos que se generan al interior del grupo (Galindo \& Parra-Valencia, 2015), nos interesamos en las experiencias grupales para el acompañamiento psicosocial en contextos de represión política y de guerra.

La importancia del grupo para la elaboración de vivencias violentas se resalta, entre otras investigaciones, en el caso del Instituto Latinoamericano de Salud Mental y Derechos Humanos (Ilas); allí se estudiaron las formas de acción grupal en El Salvador, Chile, Argentina y Guatemala, durante la década de los años 90. Su labor se basó en la perspectiva del trauma psicosocial propuesto por Ignacio Martín-Baró (1990, p. 78), que enfatiza en la herida provocada por la guerra, que implica «un carácter dialéctico»; es decir, que «se alimenta» de la relación entre individuo y sociedad.

El equipo del Ilas trabajó desde los procesos grupales, con técnicas creativas como el taller, el juego dramático y el psicodrama, con recursos gráficos y plásticos, narración, y también con los grupos psicoterapéuticos breves psicodinámicos. Se propusieron algunas respuestas terapéuticas preventivas-educativas para las jóvenes y los jóvenes de los contextos de represión con quienes se trabajó, orientadas al desarrollo de redes y pertenencias grupales. Esta investigación-acción nos aporta, en palabras de los psicólogos David Becker, Germán Morales y María Inés Aguilar (1994, p. 153), una comprensión de la función grupal como espacio de contención y elaboración.

\section{Arte y jóvenes}

«El arte, el circo y el encuentro con el otro, se han convertido en el escenario perfecto, donde él como joven puede existir, exponer, reconfigurar constantemente sus propias identidades»

(Romero, 2012).

Con respecto al papel del arte para la gente joven, una serie de estudios nutrieron nuestra investigación, dando cuenta de que las personas jóvenes transforman escenarios de violencia a través de estrategias artísticas y culturales. Lo artístico ha sido clave en las expresiones juveniles y de esta idea dan cuenta diferentes investigaciones que se interesan, como nosotras, en la relación entre el arte y los individuos jóvenes.

Janeth Restrepo-Marín (2013) investigó expresiones juveniles en contextos de violencias en Medellín. Identificó varias características en común, entre otras la identidad colectiva a través del vínculo con el otro, donde se generan diálogos desde las expresiones artístico-culturales al interior de los barrios, que permiten promover una cultura de paz por medio de acciones sociales. Sin duda, estas estrategias nacidas de los sujetos jóvenes se configuran en formas de hacer memoria y denunciar el olvido, como resistencia a la violencia y al conflicto prolongado que ha permeado a la ciudad durante las últimas décadas.

También en Medellín, López (en Torres et al., 2012, p. 94) resalta que las personas jóvenes «transmutan» las vivencias dolorosas ocasionadas por la violencia, en creatividad, ejercicios de reflexión y expresiones culturales, como proceso de sanación y reconfiguración colectiva.

Por su parte, la Corporación Vínculos (2011) menciona como estrategia de resistencia el trabajo realizado por la Casa de la Juventud de Tambo en el departamento del Cauca, señalando la importancia de la reactivación de las organizaciones juveniles desintegradas por la guerra y la violencia. Es así como la iniciativa de la Casa de la Juventud de Tambo, por medio de actos lúdicos y culturales —como cine al parque y campeonatos de futbol- , fomentaron la recuperación de la confianza en la gente habitante de la comunidad, con el objetivo de disminuir el temor sembrado por el conflicto armado y reconstruir el tejido social. Así mismo, promovieron el surgimiento de un modelo pedagógico 
alternativo de educación formal, conocido como «Maestra de vida», para prevenir la vinculación de niños, niñas y jóvenes a grupos armados.

Por otro lado, la publicación de la cooperación alemana (Le Blanc, 2014), Cercapaz (Cooperación entre Estado y Sociedad Civil para el Desarrollo de la Paz), visibilizó la existencia de catorce iniciativas juveniles de construcción de paz en el departamento de Caldas, Norte de Santander y Cesar. En el estudio se reconoce el trabajo de dichas iniciativas en cada uno de los territorios; se resalta que las jóvenes y los jóvenes logran contrarrestar los diferentes tipos de violencia a través de saberes, proyectos y estrategias colectivas, desde visiones artísticas, educativas, pedagógicas y sociales; de tal forma que el arte se instaura como un elemento trascendental para los individuos jóvenes, en un espacio de incidencia y de participación juvenil.

En síntesis, conocer sobre el trabajo grupal en la gente joven nos lleva a concluir que en su caso lo grupal está estrechamente vinculado a las artes.

\section{Metodología. Una ruta para estudiar el ámbito grupal juvenil}

La metodología que utilizamos para la investigación es de corte cualitativo (Creswell, 2007) con un enfoque hermenéutico-interpretativo (Ángel, 2011). Empleamos dos estrategias para la producción de información (González-Rey, 2000), principalmente: la primera es la revisión teórica y documental, y la segunda, el trabajo de campo a partir de la aproximación a una iniciativa juvenil.

Centramos la revisión documental en aquellas investigaciones sobre: a) el acompañamiento psicosocial en el contexto de la guerra y la represión política, en Latinoamérica y Colombia; b) El surgimiento de los movimientos sociales juveniles en Colombia; c) Las perspectivas psicológicas del grupo (tradicional, individualista, interaccionista, psicoanalítica y latinoamericana); d) El significado del grupo para las jóvenes y los jóvenes; y e) Los estudios sobre las artes y lo artístico en las personas jóvenes.

La revisión incluyó fuentes de documentación primaria y secundaria, la mayoría proveniente de libros, artículos de revista y tesis. Revisamos las plataformas virtuales de publicación de artículos científicos de Redalyc, Dialnet, Scielo y Proquest, por un criterio de pertinencia en relación con el tema, y de acceso.

La segunda estrategia de la investigación —el trabajo de campo- consistió en el acercamiento a la iniciativa juvenil Laboratorios de Paz, que detallamos a continuación.

\section{Acercamiento a una iniciativa juvenil, desde la observación de bebés}

Del corazón joven de la localidad de Suba, parte del Distrito Capital de Bogotá, surge Laboratorios por la paz. Un grupo de jóvenes que realiza acciones sociales por esa paz tan anhelada en Colombia decide por ellos mismos organizarse en torno a esta iniciativa. Las personas jóvenes de este colectivo le apuestan a la concientización social y a la formación desde la música, el graffiti y el rap.

Para conocer esta experiencia y su funcionamiento psíquico grupal, nos acercamos al colectivo en diferentes momentos y escenarios en los que interactúan, a lo largo de un año. A partir de los seis encuentros que mantuvimos en total con este grupo de jóvenes, identificamos la importancia de la dimensión artística para el colectivo y sus demandas sociales y políticas.

Nuestras herramientas metodológicas fueron la conversación informal (Delgado, \& Gutiérrez, 1999). También algunas estrategias clínicas derivadas de la corriente del psicoanálisis grupalista inglés, relacionadas en particular con el uso de la técnica de observación de bebés, como la escucha flotante y la asociación libre. Para profundizar se puede consultar a Donald Winnicott (1998) y a Hilda Botero (2008), psicoanalista que introdujo la observación de bebés en Colombia.

Usamos también como herramienta metodológica los protocolos de registro de la observación psicoanalítica. 
La técnica psicoanalítica poskleiniana (Bleichmar, \& Leiberman, 1997) de observación de bebés nace en el reconocido Tavistock Institute de Londres, a principios del siglo XX. Jugó un papel fundamental en el desarrollo de la Teoría de las relaciones objetales, centrada en la importancia de los vínculos tempranos en la estructuración del psiquismo humano. Además de la observación de bebés, también es conocido por el trabajo psicoterapéutico con niños y jóvenes.

Su uso permite reflexionar acerca de las interacciones, los estados mentales y las emociones que se configuran en aquellas experiencias donde se haya gestado una relación o un vínculo (Garciandía \& Torres, 2000) en la acepción psicoanalítica del concepto. Recordemos que la observación de bebés generalmente se remite al registro de las interacciones y modos de vinculación entre bebé y quien ejerce las funciones maternales de cuidado (López \& Schnitter, 2010).

En nuestro caso, la aproximación a la iniciativa grupal juvenil de Laboratorios por la paz, estuvo inspirada en la observación de bebés, aunque en esta oportunidad la utilizamos para la observación de jóvenes, en cuanto que enmarcó la observación del funcionamiento psíquico grupal que configuran y favoreció la comprensión de cómo se relacionan los individuos jóvenes.

El criterio para la aproximación a la iniciativa juvenil estuvo en relación con una condición de grupo, con la presencia de la expresión artística y con la disponibilidad de las personas jóvenes para la observación.

Fue así como los sujetos jóvenes de Laboratorios por la Paz aceptaron nuestra propuesta de acercamiento desde la observación, en este caso clínica, a diferentes momentos y escenarios en los que compartían como grupo. Estos tuvieron lugar en la comunidad, en el barrio y en sus espacios públicos.

Se acordó un espacio académico donde el Grupo Beat Flow Music (La BFM), perteneciente a Laboratorios por la Paz, compartió su experiencia con música, grafiti y encuentro, el cual se materializó en el Foro Jóvenes, grupo y arte, que tuvo lugar en mayo de 2016 en la ciudad de Bogotá, a propósito de la presentación pública de la modalidad de grado en Psicología, de las estudiantes auxiliares de investigación, coautoras del presente artículo.

La estrategia de análisis de la información fue hermenéutico-interpretativa y siguió el procedimiento de la codificación de la Teoría Fundamentada (Ángel, 2011) para la construcción categorial, que abordamos a continuación.

\section{Discusión}

El interés en torno al grupo y al arte contemporáneo en la reconfiguración de experiencias en jóvenes, nos permitió la construcción de cinco categorías que proponemos para la discusión. Las categorías que emergieron son: Si el grupo es capaz de movilizar su mundo interior, llegará a reconfigurar su mundo exterior; el grupo protege, contiene, cura y sana; la unión y el arte como medio de reconstrucción; grupos juveniles que entretejen vínculos y sueños; $y$, por último, resistencia y grupo.

\subsection{Si el grupo es capaz de reconfigurar su mundo interior, llegara a movilizar el mundo}

\section{Hoja en blanco}

Frente a ustedes me revelo, con rimas que traen veneno.

Considero a mi enemigo, al que somete a mi pueblo.

Con miserables pagos y en su comida veneno.

Desde hoy declaro el duelo de ricos contra mi pueblo.

Ahora empuñaré mis armas, un esfero y un cuaderno.

Acompañadas de un micro y un buen sonido en estéreo.

Para darle hoy la pela, a su maldito noticiero. 
Y, como una hoja blanca, empecemos desde ceros.

(Canción. Grupo Beat Flow Music

-BFM-Laboratorios por la Paz)

Partimos del hecho de que lo grupal implica experiencias emocionales profundas y demás singularidades de la subjetividad de quienes interactúan en el grupo. Este intercambio se suma al hecho de compartir un ideal colectivo, lo que lleva a que se gesten procesos de reconfiguración subjetiva e intersubjetiva. La emergencia de este ideal colectivo es posible por aquello que el psicoanalista francés Rene Kaës (1995) nombró como aparato psíquico grupal; resultado del encuentro de los psiquismos individuales que posibilita la construcción de una realidad interna grupal, que a su vez contiene una dimensión externa al grupo.

Esta situación hace posible un intercambio recíproco entre los elementos inmersos en la realidad psíquica individual, para el establecimiento de un objetivo común que se construye desde el sujeto y su comunidad. Esta idea permite repensar el ambiente social en el que se desenvuelve.

Ahora bien, entendemos que gracias a los procesos psíquicos identificatorios que emergen entre los individuos jóvenes, es posible impulsar iniciativas que le apuesten a la transformación social. También compartimos la idea de que cada sujeto se construye a partir de los resultados de sus relaciones interpersonales, y de la presión que ha ejercido su vida psíquica. No en vano, las corrientes de la grupalidad psicoanalítica (Parra-Valencia, 2017) conciben que el grupo constituye el espacio donde sus miembros ponen en el otro, figuras que corresponden a sus fantasías inconscientes. Es decir, que ciertos aspectos inconscientes individuales se proyectan a los miembros en el grupo; así, cada integrante representa distintas partes yoicas (Aponte-Muñoz \& Dueñas-Manrique, 2016).

A la vez que los grupos mueven la vida psíquica individual, también movilizan utopías. En este sentido, consideramos como una forma creativa, aquella que encontraron las personas jóvenes de Laboratorios por la paz, para transmitir de manera simbólica un mensaje de paz, gestado desde el interior. El medio fueron dos emblemas para contrarrestar la violencia: el esfero y un cuaderno. Este simbolismo facilita que su emoción pueda ser expresada a través de estos objetos que adquieren un significado que contribuye a sembrar la esperanza, y se constituyen en una herramienta para la expresión y la composición de las canciones de la gente joven. 2015

Figura 2. Jóvenes de La BFM - Laboratorios por la Paz-, cantando en el Piloto Festival de

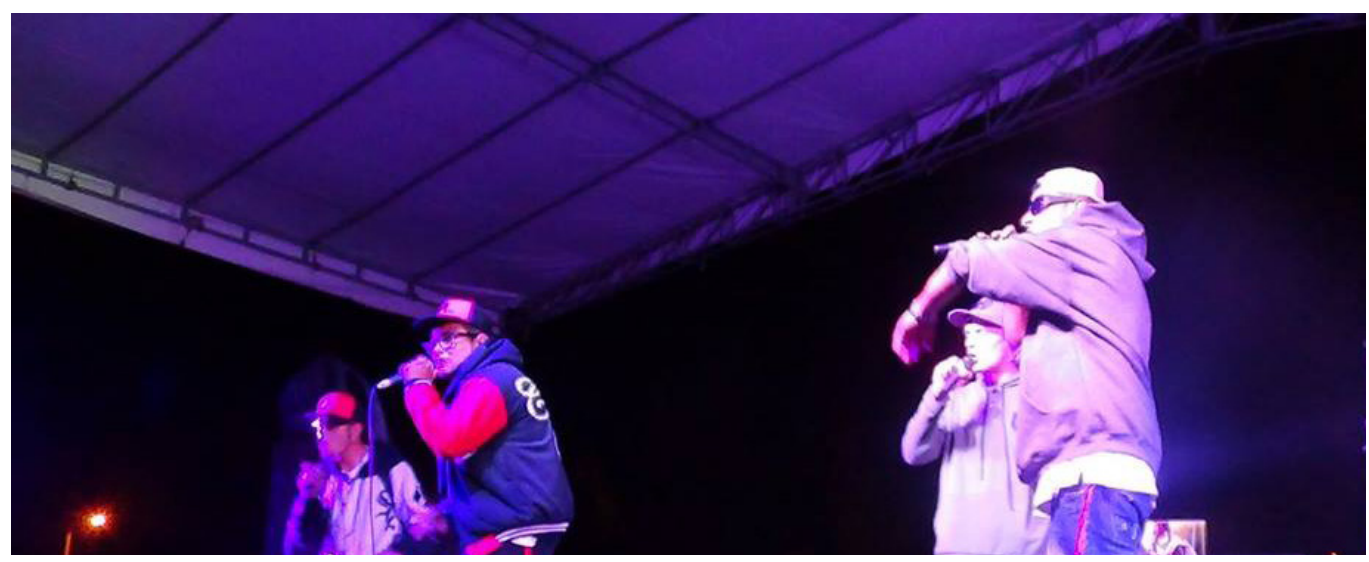

Foto: Diego Ayala.

Por otro lado, la identificación con los pares es primordial en la construcción de aquel deseo compartido de cambio. El fragmento de la canción Hoja en blanco compuesta por los sujetos jóvenes miembros de la organización Laboratorios por la Paz, es un ejemplo de la manera como las personas jóvenes han interiorizado el deseo de protestar, ante aquello considerado socialmente injusto. 


\subsection{El grupo protege, contiene y sana}

El grupo es mediador y cuenta con la capacidad de contener los estados emocionales de sus miembros. Al parecer, el grupo de jóvenes construye una capa protectora - en el sentido del pensamiento del psicoanalista francés Didier Anzieu (1998) — que sana y re-significa las experiencias dolorosas individuales, a través de acciones colectivas. Esta capa o especie de envoltura, tiene dos caras: una de ellas simboliza la realidad externa, y la otra cara es la realidad interna, donde se albergan emociones que desean ser externalizadas. Esta envoltura permite crear una representación del grupo en el sujeto, a partir de los contenidos inconscientes individuales y del diálogo con otros psiquismos en un determinado contexto.

El grupo facilita el espacio para transformar experiencias dolorosas de cada uno de los individuos participantes y de sus contenidos inconscientes. Crea un diálogo entre psiquismos, donde ninguno se percibe como un agregado al grupo, sino que el grupo es un todo que busca un objetivo: la transformación del contexto en el cual se encuentra.

No obstante, cabe mencionar que la envoltura psíquica referida es la que resguarda, contiene y define al grupo. Esto, sin recurrir a un líder que sostenga al grupo, puesto que la labor grupal esta direccionada desde un ideal común de cambio, tanto del mundo interno como externo de los sujetos. Busca que la sociedad en general sea partícipe activo de sus intervenciones y que a la vez interiorice el mensaje.

Además, conviene tener en cuenta que la labor de las iniciativas está mediada por el apoyo mutuo. Seguimos la idea de Beristain y Riera (1993) al entender el apoyo mutuo como la capacidad que tienen los miembros de contener mutuamente reacciones impulsivas, compartir experiencias y llevar a cabo soluciones a los problemas.

En las iniciativas de las personas jóvenes, evidenciamos que todos los actores son fundamentales y que no existen jerarquías. Además, en varias oportunidades las jóvenes y los jóvenes realizan actividades de inclusión de la comunidad. Aquí no solo se entiende el sentir propio, sino que además se piensa y se considera a los otros. La apertura de espacios para talleres, conversatorios y demás actividades, invita a la reconstrucción de la memoria y a la reconfiguración del sentir de la comunidad.

Beristaín plantea que el apoyo mutuo es posible en la medida en que al interior de los grupos se fomenten tres principios básicos: La igualdad, entendida como un nivel humano en el que todas las personas son miembros del grupo; la aceptación, que consiste en respetar a las personas con sus sentimientos y pensamientos; y la comprensión, la cual es descrita como la capacidad de ponerse en el lugar del otro.

El apoyo mutuo en el grupo favorece los procesos terapéuticos en los individuos jóvenes, y en aquello que sana. Es decir, el grupo representa para las personas jóvenes un potencial de alternativas de apoyo que les permite mantener las ilusiones y el deseo de cambio.

\subsection{El arte como medio para la reconfiguración y la unión}

Para los sujetos jóvenes de Laboratorios por la paz, la realización en grupo de murales y composiciones musicales se constituye en un medio que les permite no solo expresar sino también metabolizar y reconfigurar emociones como la rabia, la impotencia, la frustración, la angustia o el miedo. Se podría decir que en el grupo es posible sublimar algunas vivencias del conflicto.

Ahora bien, los grupos juveniles en algunos casos funcionan dentro de un encuadre particular, dado que son personas reunidas en determinado espacio, con horarios de tiempo establecidos de común acuerdo; allí se instaura una serie de condiciones comunes que permiten el encuentro y la organización necesaria para alcanzar objetivos en común - desde iniciativas con las que se comprometen-, referidos a la transformación y la construcción de paz. Allí, las jóvenes y los jóvenes fomentan las artes como herramienta para las metas compartidas. 
Por otro lado, es posible entrever que las acciones artísticas se ubican en el lugar de aquello que Enrique Pichón-Rivière (citado por Buzzaqui, 2008) denominó la tarea primaria, como la meta en común del grupo: aquello que los une.

«La tarea es la marcha del grupo hacia su objetivo, es un hacerse y un hacer dialéctico hacia una finalidad, es una praxis y una trayectoria» (Pichón-Rivière, citado por Buzzaqui, 2008, p. 189).

De la misma forma, retomando la experiencia con Laboratorios por la paz, es posible identificar un grupo sofisticado o un grupo de trabajo, como lo denominó el psicoanalista inglés Wilfred Bion, en cuanto a que esta iniciativa da cuenta del anhelo compartido de construir una realidad social diferente. Es decir, que el grupo representa para las personas jóvenes un potencial de alternativas de apoyo que les permite mantener las ilusiones y el deseo de cambio, a partir de la unión. Podría decirse que han superado los estados emocionales de angustia, odio y destrucción, desde el deseo del grupo por fomentar las expresiones artísticas como herramienta de cambio, donde los individuos jóvenes comparten ideales; incluso sin la necesidad de un único líder que mantenga la unidad del grupo.

Figura 3. Realización del mural «Educarte» durante un taller de formación con jóvenes pertenecientes al barrio Villa María-Suba



Foto: Angie Carolina Aponte-Muñoz.

Por ejemplo, el grafiti para las personas jóvenes de Laboratorios por la paz representa un medio de comunicación y una alternativa de hacer presencia en los barrios. Es allí donde la expresión artística logra transmitir un deseo colectivo y facilitar los procesos de sublimación.

\subsection{Los grupos juveniles entretejen vínculos y sueños}

"Contágiate de paz», fue el lema testigo de que los sueños que se tejen con el otro son posibles, como aquella tarde del sábado 28 de noviembre de 2015, durante el Piloto Festival por la paz. El encuentro fue organizado por Laboratorios por la paz, y por otros colectivos como Redvolución, Rutas por la Paz y Ceta. El Piloto Festival es la muestra de que la fragmentación y la polarización social (Parra-Valencia, 2008, p. 275) de sectores y barrios, no representó un impedimento para que varios grupos y colectivos decidieran unirse; lo cual configura su derecho a la libre circulación y a la vinculación libre con sus pares, esta vez con el objetivo de contribuir a las apuestas por una cultura de paz con la que sueñan, como lo menciona un joven rapero al cierre del Festival: "Todos seremos una fuerza imparable que luchará con la fuerza de la libertad».

El grupo tiene la capacidad de canalizar las necesidades personales, ya que se condensan como un interés colectivo, como un sueño (Martín-Baró, 1990). Aunque algunas veces los grupos se constituyan por intereses individuales, la mayoría de veces estos se reorientan a sueños colectivos.

Entonces, el grupo constituye para el sujeto joven un lugar para estar en comunión con otros y 
agruparse en torno a un anhelo en común, que va más allá de los propios intereses. En el caso del Piloto Festival, el interés colectivo fue manifestar un mensaje de oposición, desde su comunidad, frente a una sociedad que ha naturalizado el conflicto social y la estigmatización de la juventud y de los grupos sociales populares.

Figura 4. Muestra realizada por un joven durante el Piloto Festival, dentro de las actividades que se ofrecen a la comunidad del barrio Villa María (Localidad de Suba)

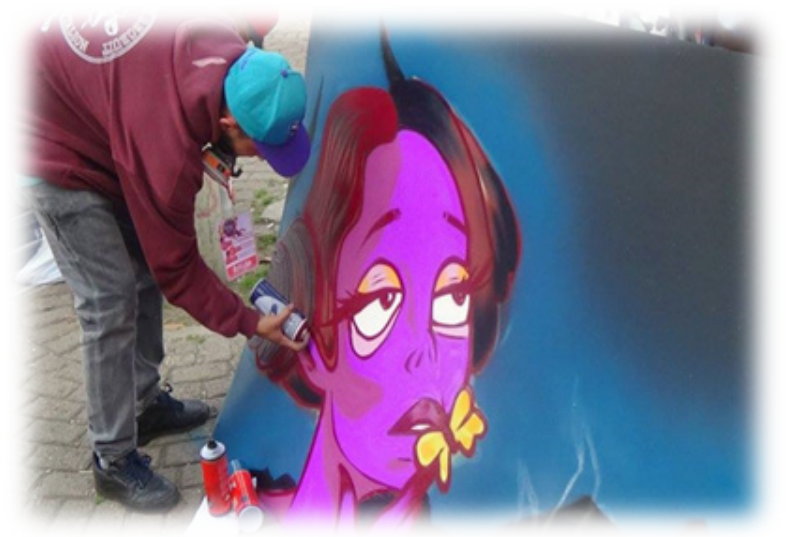

Foto: Diego Ayala.

Ahora bien, a pesar de que estos individuos jóvenes pertenecían a colectivos diferentes, lograron construir un espacio para tejer vínculos sociales, comunitarios y barriales, donde hubo lugar para los anhelos de todos, más allá de las diferencias y particularidades de cada una de las iniciativas juveniles.

\subsection{Resistencia y grupo}

En esta última categoría encontramos que el surgimiento de los grupos adquiere un significado que trasciende al individuo; aquí los mensajes de protesta y rechazo hacia una sociedad injusta, no se logran de manera individual sino colectiva.

En el grupo emergen un sinfín de caminos que abre posibilidades de resistencia y cambio, como aquellas acciones de empoderamiento de diversos colectivos y organizaciones en diferentes territorios del país.

De acuerdo con lo anterior, la conformación de un grupo requiere de un proceso de transformación individual, puesto que como expone Felipe Pinto (2010), cuando el individuo hace parte de un grupo en sus inicios, mantiene el deseo de conservar su individualidad y diferenciarse de los demás miembros; pero esto deja de suceder con el surgimiento de la grupalidad (Galindo \& Parra-Valencia, 2015).

La iniciativa de la Red Juvenil de Medellín, creada a finales de los años 80, tiene como objetivo visibilizar el papel protagónico, crítico y propositivo de la gente joven frente a los problemas colombianos. A través de propuestas y prácticas de acción política de No violencia Activa (Nova), se resisten a la guerra. Estos individuos jóvenes han creado planes estratégicos, tales como «haciendo resistencia juvenil en contextos de guerra», talleres sobre objeción de conciencia, participación y empoderamiento juvenil en derechos humanos.

Esta iniciativa se relaciona con lo que postuló Anzieu acerca del surgimiento de grupos en oposición a la sociedad, esperando que un día logren cambiar aquello que prohíben las mayorías; donde soñar se convierte en el primer paso, pues es desde la vida fantasmática del grupo - construcción de fantasías colectivas - donde surge el actuar del grupo que direcciona sus propuestas y motiva su unidad.

En el siguiente fragmento musical se da cuenta de lo mencionado en el párrafo anterior, donde el objetivo de las jóvenes y los jóvenes es transmitir un mensaje de resistencia colectivo. 
"Lo que buscamos desde el hacer valer y comprender que hoy el poder (arriba, arriba) del pueblo colombiano, lo que buscamos es hacer valer, saber porque al parecer (súbala, súbala, porque nos vamos) lo que buscamos no es el poder lo que buscamos, reventar entera y romper esas cadenas, la unión, la fuerza»

(Versos de una composición de la BFM, Laboratorios por la paz)

\section{Conclusiones}

La revisión documental y el acercamiento a la iniciativa juvenil Laboratorios por la paz, nos permitió visibilizar que las jóvenes y los jóvenes generan conciencia de cambio, a través del vínculo que se vive con la otra y el otro. Más allá de una mirada patologicista que solo se centra en los traumas desencadenados por la violencia, invitamos a las disciplinas psicológicas y a las demás ciencias sociales, a acercarse desde otro lugar a los grupos juveniles que nacen y se mantienen en lo local y en diferentes territorios urbanos y rurales.

Esta investigación es la muestra de que las instituciones estatales no son las únicas promotoras de un cambio social. Argumentamos que las propuestas de las personas jóvenes desde sectores vulnerados, generan procesos reflexivos y alternativos que inciden de manera positiva en las comunidades, a partir de sus formas particulares de ser, actuar y sentir.

Nuestra postura investigativa se centra en el punto de vista de los individuos jóvenes, en particular desde aquellas voces que no son escuchadas pero que participan de las expresiones artísticas donde los sujetos jóvenes nos transmiten que existen, resisten y grupalmente transforman escenarios locales.

Enfatizamos en la comprensión particular de las lógicas y estrategias que se gestan al interior de cada grupo para la reconstrucción del tejido social y comunitario compartido, en cuanto a que cada grupo tiene diferentes formas de organizarse y de generar procesos de apoyo mutuo, de cohesión y de transformación.

Para finalizar, buscamos dejar abierta la posibilidad de que el quehacer psicosocial profundice en lo grupal y en su potencial para fomentar los sueños y las esperanzas. Esta invitación destaca los procesos de reconfiguración grupal y por ende individual, en la conformación de un grupo protector.

También, resaltamos la unión con la otra y con el otro que se logra en el grupo, como lugar de reflexión, transformación y cura.

\section{Lista de referencias}

Ángel, D. (2011). La hermenéutica y los métodos de investigación en ciencias sociales. Estudios de Filosofia, 44, 9-37.

Anzieu, D. (1998). El grupo y el inconsciente lo imaginario grupal. Madrid: Biblioteca Nueva. Aponte-Muñoz, A. C., \& Dueñas-Manrique, M. M. (2016). Jóvenes, grupo y arte. Investigación,

Psicología e iniciativas sociales de paz en Colombia. (Tesis de Psicología). Universidad Cooperativa de Colombia, Bogotá, D. C., Colombia.

Becker, D., Morales, G., \& Aguilar, M. (Eds.) (1994). Trauma psicosocial y adolescentes latinoamericanos: formas de acción grupal. Santiago de Chile: Instituto Latinoamericano de Salud Mental y Derechos Humanos (Ilas).

Beristain, C. M. (2010). Manual sobre perspectiva psicosocial en la investigación de derechos humanos. Bilbao:Hegoa.

Beristain, C., \& Riera, F. (1993). Afirmación y resistencia: la comunidad como apoyo. Bacerlona: Virus Editorial.

Bion, W. (1997). Experiencias en grupo. Buenos Aires: Paidós.

Bleichmar, N., \& Leiberman, C. (1997). El Psicoanálisis después de Freud. Buenos Aires: Paidós.

Botero, H. (2008). ¿Qué es la observación de bebés? Algunas consideraciones. Psicoanálisis, 20(2), 103-124. 
Buzzaqui, A. (2008). El grupo operativo de Enrique Pichón-Rivière: análisis y crítica. (Tesis Doctoral). Universidad Complutense de Madrid, Madrid, España.

Cinep, \& Justicia y Paz. (1998). Banco de datos de derechos humanos y violencia política, Revista Noche y Niebla, 10, 1-107.

Corporación Vínculos. (2011). Niños, niñas y jóvenes en riesgo. Bogotá, D. C.: Anthropos.

Creswell, J. W. (2007). Qualitative inquiry and research design: Choosing among five approaches. Los Ángeles: Sage Publications.

Delgado, J. M., \& Gutiérrez, J. (Coords.) (1999). Métodos y técnicas cualitativas de investigación en ciencias sociales. Madrid: Síntesis.

Galindo, D., \& Parra-Valencia, L. (2015). Grupalidad: un camino al lado de los otros como potencial de sanación psíquica. Tesis Psicológica, 10(1), 132-143.

Garciandía, J., \& Torres, N. E. (2000). Vínculos 1. Aspectos generales y vínculo de amor. Bogotá, D. C.: Impresión Javegraf.

González-Rey, F. (2000). Investigación cualitativa en Psicología: rumbos y desafíos. México, D. F.: International Thomson Editores.

Grinberg, L., Langer, M., \& Rodrigué, E. (1974). Psicoterapia del grupo: su enfoque psicoanalítico. Buenos Aires: Paidós.

Kaës, R. (1995). El grupo y el sujeto del grupo: elementos para una teoría psicoanalítica del grupo. Buenos Aires: Amorrortu.

Le Blanc, J. (2014). Iniciativas con jóvenes en prevención de violencias y construcción de pazaprendizajes y recomendaciones para la práctica y la decisión política. Bogotá, D. C.: Cercapaz.

López, M. (2012). Reflexiones en torno a la intervención e interacción con jóvenes en conflicto armado urbano, en la comuna 13 de Medellín. Reflexiones contemporáneas sobre la intervención e interacción sociocultural. Medellín: Escuela de Animación Juvenil.

López, M. I., \& Schnitter, M. (2010). Matriz de relación primaria en casos de niños y niñas con problemas de aprendizaje. Revista Latinoamericana de Ciencias Sociales, Niñez y Juventud, 8(2), 1099-1116.

Martín-Baró, I. (1990). Psicología social de la guerra: trauma y terapia. San Salvador: UCA Editores.

Parra-Valencia, L. (2008). Introducción a la psicología de la guerra. El Ágora. Revista de La Universidad San Buenaventura, 8(2), 269-280.

Parra-Valencia, L. (2014). Prácticas y experiencias colectivas ante la guerra y para la construcción de paz. Iniciativas sociales de paz en Colombia. El Ágora, 14(2), 377-398.

Parra-Valencia, L. (2016). Acompañamiento en clínica psicosocial: una experiencia de investigación en tiempos de construcción de paz (Colombia). Bogotá, D. C.: Cátedra Libre.

Parra-Valencia, L. (2017). Clínica psicosocial. Encuentro entre Psicología Comunitaria y Psicología Clínica. "Material de apoyo docente (inédito)". Programa de Psicología. Bogotá, D. C: Universidad Cooperativa de Colombia.

Pinto, F. (2010). Grupalidad y mecanismos de defensa en el grupo. Santiago de Chile: Universidad de Chile, Facultad de Ciencias Sociales, Carrera de Psicología.

Restrepo-Marín, J. (2013). Expresiones juveniles en espacios de violencias: una forma de hacer memoria y denuncia el olvido. Revista de Ciencias Sociales. Universidad San Buenaventura, 11(2), 232-489.

Rolnik, S. (2001). ¿El arte cura? Conferencia en el contexto de los debates Arte, locura y cura. Macba. Barcelona. Quaderns portàtils, 02, 1-8.

Romero, A. (2012). Sujetos políticos y actores sociales: la configuración de las identidades de los y las jóvenes de la localidad Rafael Uribe Uribe en la ciudad de Bogotá. Bogotá, D. C.: Pontificia Universidad Javeriana.

Torres, A., Ghiso, A., Castro, C., López, M., Viché, M., Sepúlveda, M., ..., Ortega, P. (2012). Reflexiones contemporáneas sobre la intervención e interacción sociocultural. Medellín: Escuela de Animación Juvenil.

Winnicott, D. (1998). Los bebés y sus madres: el primer diálogo. Barcelona: Paidós.

Rev.latinoam.cienc.soc.niñez juv 16 (2): 853-865, 2018

http://revistalatinoamericanaumanizales.cinde.org.co

doi:https://doi.org/10.11600/1692715x.16214



